\title{
Actitudes hacia la lectura, sentimiento de competencia, implicación familiar y comprensión lectora en segundo curso de Educación Primaria
}

\section{Ángeles Melero ${ }^{1}$, Ruth Villalón ${ }^{1}$ y Belén Izquierdo- Magaldi $^{1}$}

${ }^{1}$ Departamento de Educación

Universidad de Cantabria

\section{España}

Correspondencia: Ángeles Melero, Universidad de Cantabria, Departamento de Educación, Avda. de los Castros, s/n 39005, Santander.E-mail: meleroma@unican.es

(C) Universidad de Almería and Ilustre Colegio Oficial de la Psicología de Andalucía Oriental (Spain)) 


\section{Resumen}

Introducción. En la lectura, instrumento cultural clave que hay que aprender a dominar en las sociedades letradas, confluyen aspectos cognitivos, afectivo-motivacionales y contextuales. Pero en el ámbito internacional hay escasez de investigaciones sobre la relación entre comprensión lectora, actitudes hacia la lectura, sentimiento de competencia e implicación familiar en los dos primeros cursos de Educación Primaria. Además, no hay estudios en España que aborden esas variables al mismo tiempo.

Método. Nuestro estudio investiga la relación entre estas cuatro variables en alumnado español de segundo curso de Educación Primaria mediante un estudio de corte cuantitativo (descriptivo y correlacional) en el que han participado 181 niños y 167 niñas.

Resultados. Se hallaron actitudes muy positivas hacia la lectoescritura así como un elevado sentimiento de competencia. Las niñas obtuvieron puntuaciones más altas que los niños. Esta última variable y la implicación familiar exhibieron correlaciones significativas con la comprensión lectora.

Discusión y conclusiones. Se resalta la importancia de trabajar en la implicación de las familias, de mantener un positivo sentimiento de competencia y de conocer las tempranas diferencias en actitudes y preferencias de niños y niñas por parte del profesorado.

Palabras Clave: Lectura, comprensión, actitudes, sentimiento de competencia, implicación familiar, género. 


\section{Abstract}

Introduction: Reading is a key cultural instrument which needs to be mastered in literate societies. It brings together cognitive, affective-motivational and contextual aspects. But there is a lack of research about the relationship between reading comprehension, attitudes towards reading, feeling of competence and parental involvement in the two first grades of Primary Education. Furthermore, there are no studies in Spain that adress these variables at the same time.

Method: This study examines the relationship between these four variables with Spanish students at the 2nd grade of Primary Education through a quantitative study (descriptive and correlational) in which 181 boys and 167 girls participated.

Results: Very positive attitudes towards reading and writing, as well as a high feeling of competence were found. Girls obtain higher scores than boys. Feeling of competence and family involvement exhibited significant correlations with reading comprehension.

Discussion and Conclusions: The relevance of fostering the involvement of families in their childrens' acquisition of reading and writing, of maintaining a positive feeling of competence is highlighted and of teachers' knowledge of differences in attitudes and preferences of girls and boys are discussed.

Keywords: Reading, Comprehension, Attitudes, Feeling of competence, Parental involvement, Gender. 


\section{Introducción}

La lectura es un importante instrumento cultural y una práctica social (Kalman, 2008) que permite a las personas aprender a lo largo de toda la vida y, en especial, poder hacerlo de manera autónoma. Comprender textos en las actuales sociedades letradas es crucial para cualquier ciudadano en el ámbito académico, profesional y personal. Su naturaleza cultural e histórica se hace patente actualmente en que su utilización se ha ampliado a gran parte de la población a medida que han aparecido nuevos dispositivos para leer y escribir. Estos, a su vez, han dado lugar a nuevos usos cotidianos que se añaden a los incorporados en el sistema escolar y laboral. En cualquiera de estos escenarios, comprender implica capacidades complejas en las que intervienen muchas variables psicológicas y contextuales que interaccionan entre sí. Refiriéndonos a las psicológicas, estas son tanto de naturaleza cognitiva como emocional o motivacional, aunque se han estudiado en mayor medida las primeras que las segundas. No obstante, en los últimos años se ha retomado el interés por estudiar estas últimas, entre las que encontramos las actitudes que las personas mantienen cuando abordan tareas de comprensión y de producción de textos (Conradi, Jang y McKenna, 2014).

Pero no solo encontramos variables personales influyentes sobre la lectura, también las hay de tipo contextual que, a su vez, influyen en esas variables personales. Dentro de las contextuales, una relevante que ha merecido una atención especial es la implicación de las familias en el aprendizaje de la lectura dentro del sistema escolar. Así pues, nuestro objetivo ha sido explorar la relación entre la comprensión lectora (CL), las actitudes hacia la lectura (AL), el sentimiento de competencia como lector (SCL) y la implicación familiar (IF) en este proceso, centrándonos en los momentos iniciales de este aprendizaje, en segundo curso de Educación Primaria.

\section{Comprensión lectora, actitudes hacia la lectura y sentimiento de competencia en lectura}

En la literatura científica, comprender un texto es mucho más que saber contar lo que en él está explícitamente dicho. Las personas nos movemos desde la capacidad de localizar y recordar información explícita (base del texto) hasta la de poder hacer inferencias a partir de lo que pone el texto (modelo de la situación) (Kintsch y Rawson, 2008; MacNamara y Magliano, 2009). Estas inferencias implican deducir e incluso elaborar información nueva que, en interacción con el texto, relacione nuestros conocimientos previos sobre diversos aspectos del mundo con lo que aparece en el discurso escrito. Además, comprender involucra la habili- 
dad para juzgar cómo está escrito el texto (su estilo y su rigor, por ejemplo) y, en general, para advertir la relación entre los aspectos formales del texto y su contenido. En definitiva, la complejidad de la comprensión lectora necesita estar sustentada no solo en procesos cognitivos básicos sino también en aspectos de naturaleza motivacional, como son las AL o el SCL, mucho más si tiene lugar en un escenario de aprendizaje descontextualizado como es el escolar, alejado de los usos sociales de la lectura.

Las actitudes son evaluaciones generales y estables que las personas elaboran sobre otras personas, ideas o tareas (Briñol, Falces y Becerra, 2007). En este sentido, juegan un papel importante en los procesos psicológicos básicos de atención, concentración y memoria. Por otro lado, determinan la motivación y el esfuerzo que las personas ponen en marcha cuando desarrollan una actividad; por ejemplo, cuando leen (Lockwood, 2012; Schiefele, Schaffner, Möller y Wigfield, 2012). Y de este modo condicionan nuestra conducta, porque pueden favorecer (o, por el contrario, dificultar) la implicación que las personas ponen en marcha al leer. A su vez, a través de estas variables pueden impactar sobre la calidad de los procesos cognitivos desplegados. Cerrando el círculo retroalimentador, si estos procesos cognitivos no provocan, al menos, un relativo éxito lector, queda abonado el terreno para que se desarrollen AL negativas (Kaniuka, 2010). Por todo ello, el papel de estas actitudes ha recibido la atención de los investigadores en comprensión lectora.

Uno de los temas estudiados es cómo evolucionan a lo largo de la escolaridad, pero se han llevado a cabo escasos estudios en los primeros cursos de Educación Primaria, justo cuando se está adquiriendo la lectura. Los que existen (Izquierdo-Magaldi, Melero y Villalón, en prensa; Merisuo-Storm y Soininen, 2014) han encontrado que el alumnado de primero y segundo curso mantiene una AL muy positiva.

Otro asunto tratado ha sido el de las relaciones entre AL y el rendimiento en tareas lectoras. En España, no obstante, hay falta de investigaciones sobre este tópico y en el panorama internacional son muy escasas, de nuevo, las que se realizan en los dos primeros cursos de Educación Primaria. Los datos de las evaluaciones internacionales de la competencia lectora nos hablan de la importancia que tiene implicarse en la lectura y disfrutar con la misma. Así, en el caso de PIRLS 2016 (Mullis, Martin, Foy y Hooper, 2017), hay diferencias de rendimiento lector entre quienes afirman gustarles mucho la lectura y quienes dicen que no les gusta leer. Por otra parte, el metanálisis de Petscher (2010) encuentra que la relación entre AL y 
ejecución lectora es moderada (rango de 0.20 a 0.40) y más sólida en Educación Primaria que en los cursos sucesivos. Posteriormente a este metanálisis, McGeown, Johnston, Walker, Howatson, Stockburn, y Dufton (2015) hallaron, con niños entre 6 y 7 años de edad (primer curso), que las AL son incluso predictoras del éxito lector, medido a través del reconocimiento de palabras. Sin embargo, otros autores no han encontrado esta relación en ese mismo curso. En el trabajo de Nurmi y Aunola (2005), con niños de primero y segundo curso, las AL positivas no se reflejaron en un mayor desarrollo de la competencia lectora. De la misma forma, Merisuo-Storm y Soininen (2014) no encuentran la relación en su muestra de segundo curso. Chapman y Tunmer $(1995,1997)$, usando una escala en la que hay mezcla de ítems de actitudes y de autoconcepto lector, tampoco habían hallado la relación en primer curso con tareas de descodificación, a diferencia de los resultados de McGeown et al. (2015), pero, en cambio, sí en segundo y tercer curso (más intensa en este último) con tareas de comprensión.

En general, podemos decir que hay una tendencia a encontrar la relación a partir de cuarto curso inclusive, pero con excepciones, y que los datos son más contradictorios respecto a los dos primeros cursos de Educación Primaria, por lo que, tal como afirman McGeown et al. (2015), son necesarios más estudios. Además, y de forma aún más evidente, faltan estudios que incluyan CL, ya que, con muy pocas excepciones, los que existen en esas edades miden solo la habilidad para la descodificación. Asimismo, escasean datos con hispanoparlantes.

Las investigaciones también han considerado el papel del SCL entre las variables motivacionales que impactan en el rendimiento lector. Este hace referencia a la opinión o creencia sobre si se tiene, o no, facilidad o competencia para entender los textos. Ha sido evaluada de manera muy dispar y, en general, bien solicitando percepciones de competencia (Normalmente leo bien), bien pidiendo percepciones de facilidad o dificultad para leer (Leer me resulta fácil) (Chapman y Tunmer, 1995). En nuestro caso, hemos optado por evaluar el SCL a través de la percepción de facilidad de la tarea, como Merisuo-Storm y Soininen (2014).

Al igual que en el caso de las AL, diversos estudios han demostrado que los estudiantes de los dos primeros cursos tienen un alto sentimiento de competencia, así como que hay una relación positiva entre esta variable y diferentes medidas de comportamiento y rendimiento lector, entre los que está de nuevo PIRLS. Así, Mullis et al. (2017) encuentran que esta variable está positivamente relacionada con el grado de CL, de modo que los escolares con mejor autoconcepto como lector son significativamente mejores lectores. En el caso de Espa- 
ña, los estudiantes con bajo SCL obtuvieron puntuaciones en CL más bajas que sus compañeros con alto SCL. Merisuo-Storm y Soininen (2014) hallan también esta relación en segundo de Educación Primaria. Y en la investigación de McGeown et al. (2015) la autocompetencia como lector (usando el mismo instrumento que PIRLS 2011) predice el éxito en una prueba de reconocimiento de palabras en niños de 6 y 7 años. Los resultados de Carroll y Fox (2017), usando una prueba de autoeficacia en lectura con niños de 8 a 11 años, señalan que correlaciona con lectura de palabras, aunque no lo hace con CL. Por su parte, Katzir, Lesaux y Kim (2009) encuentran que, sobre todo la puntuación obtenida en las preguntas sobre facilidad autopercibida para la lectura elaboradas por Chapman y Tunmer, no solo correlaciona, sino que explica el rendimiento en reconocimiento de palabras y en CL en estudiantes de cuarto curso de Educación Primaria.

Respecto a las diferencias entre niños y niñas en AL, no son numerosas las investigaciones sobre ello, y menos aún que cuenten con participantes de los dos primeros cursos de la escolaridad. De las investigaciones con participantes de la misma edad que los de la presente (7-8 años), encontramos que la de McGeown et al. (2015) arrojó diferencias significativas ( $\eta^{2}$ parcial $=0.08$, que corresponde a un $d=0.6$, un tamaño del efecto medio) al igual que, en nuestro país, la de Artola, Sastre y Alvarado (2018) $(d=0.45)$. Sin embargo, no las hallaron Merisuo-Storm y Soininen (2014) en la puntuación total del cuestionario utilizado, aunque sí en algunos de los ítems. Así pues, en la edad en la que se sitúa esta investigación, los resultados no son plenamente coincidentes. En el resto de cursos (tercero a sexto) de Educación Primaria, el resultado habitual es encontrar diferencias en AL, siempre a favor de las niñas (Becker y McElvany, 2018; Logan y Johnston, 2009; McKenna, Conradi, Lawrence, Gee y Meyer, 2012; Petscher, 2010) y, asimismo, también en otros aspectos del ámbito motivacional, como motivación intrínseca, interés o valor concedido a la lectura (Kikas, Pakarinen, Soodla, Peets y Lerkkanen, 2018; Marinak y Gambrell, 2010; McGeown, 2015; McGeown, Goodwin, Henderson y Wright, 2012; Stutz, Schaffner y Schiefele, 2016). Incluso hay datos de que estas diferencias son más amplias en lectura que en matemáticas y ciencias (McGeown y Warhurst, 2019). Cuando los estudios analizan la relación entre sexo y sentimiento de competencia o de autoeficacia como lector, la mayoría de los resultados no arrojan diferencias entre los dos sexos (Becker y McElvany, 2018; McGeown et al., 2015; Olivares, Fidalgo y Torrance, 2016). Sin embargo, este no es lo obtenido en el estudio de Artola et. al (2018), que sí las encuentran, a favor de los niños, por lo que tampoco en SCL hay una coincidencia total. Refiriéndonos a nuestro país, las AL también se han estudiado en sexto curso (Artola, Sastre y Barraca, 2017) 
y los resultados de nuevo encuentran que las niñas tienen puntuaciones superiores en esta variable, pero no en SCL.

La implicación familiar y su impacto en el rendimiento lector y en las actitudes hacia la lectura

No hay una definición consensuada sobre qué es implicación familiar (IF). Por un lado, hay estudios que manejan un concepto global (LaRocque, Kleiman y Darling, 2011) y, desde este punto de vista, podemos decir que consiste en la participación activa de los padres en los procesos y experiencias escolares de sus hijos (Castro, Expósito-Casas, López-Martín, Lizasoain, Navarro-Asencio y Gaviria, 2015; Jeynes, 2005). Por otro lado, hay quienes usan un concepto específico, descomponiéndolo en dimensiones, relacionadas con las expectativas familiares sobre la escuela y el tipo de prácticas lectoescritoras en el hogar, entre otros aspectos (Xu, Benson, Mudrey-Camino y Steiner, 2010; Mol y Bus, 2011; Wilder, 2014). Respecto a la forma de medir la IF, se ha acudido a informes de padres, de profesores y, si tienen edad suficiente, de los propios niños (Desforges y Abouchaar, 2003). En nuestro caso, hemos pedido a los docentes que hicieran una evaluación global del compromiso familiar con el aprendizaje lector que sus hijos estaban llevando a cabo en la escuela.

La influencia positiva de la IF sobre el rendimiento escolar general se manifiesta ya desde Educación Infantil y se va haciendo más importante durante los años de la educación obligatoria (Fantuzzo, McWayne, Perry y Childs, 2004; Galindo y Sheldon, 2012). Es independiente -según la metasíntesis de Wilder (2014)- de la definición que se adopte de IF y es moderada (Mol y Bus, 2011; Castro et al., 2015). También aparece, específicamente, sobre el rendimiento lector. Lo confirman tanto el trabajo de Kloosterman, Notten, Tolsma y Kraaykamp (2010) desde segundo a sexto curso, como el metanálisis de Sénéchal y Young (2008) desde Educación Infantil hasta tercero de Educación Primaria, así como la revisión de Van Voorhis, Maier, Epstein y Lloyd (2013), en edades de 3 a 8 años. En nuestro país, el estudio de Mora-Figueroa, Galán y López-Jurado (2016) con niños de primero de Educación Primaria con dificultades lectoras llega a la misma conclusión. No hemos encontrado en España más investigaciones que aborden la relación específica con CL en los dos primeros cursos de Educación Primaria. En el panorama internacional, cuando se han incluido estos cursos, la relación frecuente es con rendimiento en tareas de descodificación (lectura de sílabas, lectura de palabras) pero no con CL. 
En cuanto a los estudios que analizan la relación entre IF y variables motivacionales, son mucho menos numerosos que los que tratan la vinculación con rendimiento y, específicamente, son escasísimos los que comprueban la vinculación entre esa variable familiar y, en concreto, las actitudes de sus hijos hacia las materias escolares o hacia algún área curricular. En una revisión de la literatura sobre IF y motivación de los estudiantes por parte de Gonzalez-DeHass, Willems, y Holbeinet, (2005) se detecta que existe una mayor motivación por la lectura si los estudiantes cuentan con padres implicados en este proceso y apoyan desde casa este aprendizaje escolar, releyendo libros o textos, por ejemplo. Por su parte, Adamski, Fraser y Peiro (2013) encontraron relación correlacional y causal entre actitudes hacia una asignatura lingüística y la implicación familiar autopercibida por estudiantes de cuarto y sexto de Educación Primaria, así como una correlación entre actitudes hacia el español y rendimiento en español. Sin embargo, no conocemos investigaciones que analicen la relación entre IF y AL (y no solo la de IF con rendimiento).

\section{Objetivos}

Dada la naturaleza exploratoria de este estudio, no hemos formulado hipótesis, pero sí una serie de objetivos específicos, que han guíado el estudio: 1) Conocer cuáles son las AL y el SCL en segundo curso de Educación Primaria. 2) Establecer si hay diferencias entre niñas y niños en CL, AL y SCL y en estas mismas variables en función del grado de implicación familiar. 3) Averiguar si existe relación entre actitudes hacia la lectura, sentimiento de competencia como lector, implicación familiar y rendimiento lector.

\section{Método}

\section{Participantes}

Participaron 348 estudiantes de segundo curso (181 niños y 167 niñas), de entre 7 y 8 años, (media de edad $=7$ años y 3 meses) y sus 31 docentes. La muestra inicial fue de 388 alumnos, pero se retiraron los datos de aquellos sujetos que no conocían perfectamente el español, así como los de quienes tenían desfase curricular, especiales problemas de aprendizaje o necesidades educativas específicas. Los participantes pertenecían a siete colegios urbanos de una Comunidad Autónoma de Cantabria, tanto públicos como concertados, a los que asistían familias con diversidad de estatus socioeconómico y sociocultural. 


\section{Instrumentos}

Para medir CL se utilizaron dos instrumentos. Uno de ellos fue una selección de textos de una prueba de CL, concretamente, las pruebas ACL (Catalá, Catalá, Mireia y Monclús, 2001). Consisten en unos pequeños textos de distinto tipo seguidos de unas preguntas de comprensión que evalúan comprensión literal, inferencial y crítica. En esta prueba los niños pueden volver a leer el texto para contestar a las preguntas. Los textos ACL que cumplimentaron fueron cinco, literarios y expositivos, todos ellos continuos, y el número de preguntas al que contestaron, de 17. Se concedió un punto por cada ítem correctamente respondido y se halló la media. Por tanto, el rango de puntuaciones va de 0 a 1.

El otro consistió en pedir al tutor que situara a cada estudiante del aula en uno de tres grupos (baja competencia lectora, competencia lectora media y alta competencia lectora), otorgando las puntuaciones de 1,2 y 3 , respectivamente.

Para medir AL y SCL utilizamos una adaptación del cuestionario de Merisuo-Storm y Soininen (2012). Se eligió este instrumento porque estaba específicamente diseñado para estudiantes de segundo curso y cubría los dos constructos que nos interesaban (AL y SCL). La adaptación consistió en el ajuste de alguno de los ítems a la realidad española y a la de su sistema educativo.

La mayor parte de los items (20) miden AL. Los participantes contestan a preguntas como las siguientes: ¿Te gusta leer?, ¿Te gusta ir a la biblioteca? Una segunda parte (con 7 items) versa sobre el SCL y está formada por ítems que valoran percepción de facilidad en tareas de lectura (¿Te ha resultado fácil aprender a leer?). Siempre son frases interrogativas, como recomiendan Chapman y Tunmer (1997), muy cortas y sencillas de comprender que tienen que ver con las prácticas lectoescritoras que los niños llevan a cabo en el contexto escolar, aunque alguna de ellas también la pueden realizar con la familia. El cuestionario presenta una escala Likert de cuatro puntos, que se plasmó en cuatro caras de osos con distintas expresiones faciales que iban desde muy contento a muy descontento, siguiendo a las autoras del cuestionario original, con el objetivo de adaptarlo a la edad de los participantes. En la escala, 1 significa que tiene una actitud muy negativa (equivale a la cara del oso más descontento), y 4 una actitud muy positiva (corresponde a la cara del oso más contento). Su rango de puntuaciones, por tanto, es de 20-80. En el caso de SCL, 1 significa que es muy bajo y 4 que es muy alto, y el rango de puntuaciones es de 7-28. 
El alfa de Cronbach fue de .86 para AL y de .77 para SCL. En ambos casos, las puntuaciones utilizadas son las medias de la escala (rango de 1 a 4 ).

Para medir implicación familiar se recurrió nuevamente a los docentes, a quienes se pidió que clasificaran a las familias en uno de estos tres grupos: baja implicación, implicación media y alta implicación en los procesos lectoescritores de sus hijos. Las respectivas puntuaciones fueron de 1,2 y 3 .

\section{Procedimiento}

En una primera fase, se realizó un estudio piloto, para ajustar la formulación de los ítems del cuestionario, así como el procedimiento de aplicación de las pruebas a los niños de estas edades. En este estudio piloto participaron 41 estudiantes (20 niños y 21 niñas) de dos aulas de segundo curso de Educación Primaria.

En una segunda fase, y una vez cerrado el cuestionario, se llevo a cabo este estudio transversal descriptivo de corte cuantitativo. Se contactó con los diversos centros escolares para obtener los correspondientes permisos (equipo directivo, tutores y familias). Una vez obtenido el permiso de las dos primeras instancias, se solicitó a las familias el consentimiento informado por escrito con un texto en el que figuraba, de forma claramente comprensible, en qué consistía la investigación, su objetivo, las tareas que tenían que llevar a cabo los niños, la persona responsable y la forma de contacto con ella, así como toda la serie de derechos que reconoce la legislación vigente para las personas participantes de una investigación.

Una vez en las aulas, dada la edad de los participantes, las investigadoras presentaron los instrumentos y se aseguraron de que el alumnado comprendiera la mecánica de contestación a las pruebas.

En el caso de las pruebas ACL, cada participante las cumplimentaba de modo anónimo y de manera completamente autónoma, a su propio ritmo y sin límite de tiempo. En el caso del autoinforme sobre AL, una investigadora iba leyendo en voz alta cada una de las preguntas, de manera que el alumnado respondía simultáneamente al mismo ítem. Las otras dos investigadoras servían de apoyo, para solucionar dudas o problemas puntuales de algún niño en particular. 
En cuanto al orden de las pruebas, primero se aplicó la de CL, sin límite de tiempo, y tras un descanso, el cuestionario de AL.

Todo el proceso de recogida de datos y tratamiento de los mismos se realizó bajo las normas éticas y la normativa legal aplicable a la investigación con seres humanos.

\section{Análisis de datos}

Para comprobar la normalidad de las puntuaciones se aplicó la prueba KolmogorovSmirnoff con la corrección de Lilliefors. El resultado nos ha exigido hacer pruebas no paramétricas.

La naturaleza descriptiva y correlacional de este estudio nos llevó a tres tipos de análisis: 1) Análisis descriptivo de las variables estudiadas (media, desviación típica, puntuación mínima y máxima, rango): comprensión lectora, actitudes hacia la lectura, sentimiento de competencia como lector, implicación familiar en los procesos de alfabetización iniciales. 2) Contraste de medias: a) por sexo en comprensión lectora, actitudes, sentimiento de competencia e implicación familiar (Mann-Whitney); b) por categoría de implicación familiar en comprensión lectora, actitudes y sentimiento de competencia (Kruskal-Wallis). 3) Correlaciones (Spearman) entre comprensión lectora, actitudes, sentimiento de competencia e implicación familiar en la muestra total y por sexo (correlación biserial por rangos de Glass).

\section{Resultados}

Respecto al primer objetivo, en las Tablas 1 y 2 pueden verse las puntuaciones obtenidas en las variables estudiadas que, en su mayoría, no tienen una distribución normal. Nuestros participantes presentan una correcta comprensión lectora en las dos medidas utilizadas (claramente más baja en el grupo de baja implicación familiar), actitudes positivas hacia la lectura y un alto sentimiento de competencia como lector, así como una adecuada implicación por parte de sus familias en el proceso lectoescritor. 
Tabla 1. Descriptivos: M: Media y (D.T.: Desviación típica); Mín: Mínimo; Máx: Máximo y Rango de las variables estudiadas en la muestra total, por sexo

\begin{tabular}{|c|c|c|c|c|c|}
\hline \multicolumn{4}{|c|}{ M. (D.T.) } & \multirow{2}{*}{ Mín-Max } & \multirow{2}{*}{ Rango } \\
\hline & Muestra Total & Niños & Niñas & & \\
\hline CL-ACL & $.66(0.23)$ & $.66(0.23)$ & $.67(0.24)$ & $0-1$ & $0-1$ \\
\hline CL-D & $2.36(0.75)$ & $2.37(0.74)$ & $2.36(0.76)$ & $1-3$ & $1-3$ \\
\hline $\mathrm{AL}$ & $3.39(0.44)$ & $3.32(0.47)$ & $3.46(0.39)$ & $1.3-4$ & $1-4$ \\
\hline SCL & $3.39(0.52)$ & $3.42(0.51)$ & $3.36(0.53)$ & $1-4$ & $1-4$ \\
\hline IF & $2.43(0.69)$ & $2.42(0.67)$ & $2.44(0.70)$ & $1-3$ & $1-3$ \\
\hline
\end{tabular}

Nota. CL-ACL: comprensión lectora medida por ACL; CL-D: comprensión lectora estimada por docentes; AL: actitudes hacia la lectura; SCL: sentimiento de competencia como lector; IF: implicación familiar; Mín-Máx: Puntuación mínima y máxima

Tabla 2. Descriptivos: M: Media y (D.T., Desviación típica) de las variables estudiadas en la muestra total, para cada categoría de implicación familiar

\begin{tabular}{lccc}
\hline & BAJA & MEDIA & ALTA \\
& M (D.T.) & M (D.T.) & M (D.T.) \\
\hline CL-ACL & $.47(0.30)$ & $.65(0.20)$ & $.70(0.20)$ \\
CL-D & $1.60(0.74)$ & $2.21(0.70)$ & $2.60(0.64)$ \\
AL & $3.42(0.46)$ & $3.38(0.42)$ & $3.38(0.47)$ \\
SCL & $3.23(0.64)$ & $3.39(0.55)$ & $3.45(0.45)$ \\
\hline
\end{tabular}

Nota. CL-ACL: comprensión lectora medida por ACL; CL-D: comprensión lectora estimada por docentes; AL: actitudes hacia la lectura; SCL: sentimiento de competencia como lector 
Respecto aL segundo objetivo, cuando se comparan niños y niñas en AL se observa que hay diferencias significativas a favor de estas $(Z=-3.003 ; \mathrm{p}=.003 ; r=.18 ; d=.33)$; en cambio, no las hay en CL, SCL e IF.

Cuando se hace la comparación en función de las tres categorías de implicación familiar no encontramos diferencias significativas en AL ni en SCL, pero sí en ambas medidas de CL (ACL: $H=23.705 ; \mathrm{p}=.000$; CL-D: $H=57.307 ; \mathrm{p}=.003$ ). En el caso de CL-ACL, los contrastes post-hoc de la categoría 1 con 2 (baja con media), 1 con 3 (baja con alta) y 2 con 3 (media con alta) arrojaron de nuevo todos ellos diferencias significativas ( 1 con $2: Z=-2.92$, $\mathrm{p}=.003, d=.25 ; 1$ con $3: Z=-4.65, \mathrm{p}=.000, d=.32 ; 2$ con $3: Z=-2.765, \mathrm{p}=.006, d=$ .17), cumpliendo con los requisitos de la corrección Bonferroni. En el caso de CL-D, los contrastes post-hoc de la categoría 1 con 2 (baja con media), 1 con 3 (baja con alta) y 2 con 3 (media con alta) también demuestran diferencias significativas ( 1 con $2: Z=-4.125, \mathrm{p}=.000$, $d=.35 ; 1$ con $3: Z=-6.825, \mathrm{p}=.000, d=.48 ; 2$ con $3: Z=-4.963, \mathrm{p}=.000<, d=.30)$, cumpliendo nuevamente con los requisitos de la corrección Bonferroni.

Con respecto al tercer objetivo, realizamos correlaciones entre las variables (ver Tabla3).

Tabla 3. Correlaciones entre las variables estudiadas (Spearman)

\begin{tabular}{lccccc}
\hline & CL-ACL & CL-D & AL & SCL & IF \\
\hline CL-ACL & 1 & & & & \\
CL-D & $.51^{* *}$ & 1 & & & \\
AL & -.03 & .04 & 1 & & \\
SCL & .06 & $.20^{* *}$ & $.50^{* *}$ & 1 & \\
IF & $.27^{* *}$ & $.42^{* *}$ & -.01 & .07 & 1 \\
\hline
\end{tabular}

Notas. ${ }^{*} \mathrm{p}<.01 ; * * \mathrm{p}<., 001$

CL-ACL: comprensión lectora medida por ACL; CL-D: comprensión lectora estimada por docentes; AL: actitudes hacia la lectura; SCL: sentimiento de competencia como lector; IF: implicación familiar 
Los resultados indican que las AL no correlacionan con ninguna de las dos puntuaciones de rendimiento lector. En cambio, SCL sí lo hace cuando la comprensión lectora es evaluada por docente $(\rho=.20 ; \mathrm{p}<.01)$ e igualmente lo hace implicación familiar, esta vez con ambas medidas de comprensión (CL-ACL: $\rho=.27 ; \mathrm{p}<.01$; CL-D: $\rho=.42 ; \mathrm{p}<.01$ ).

La magnitud de la relación entre sexo y el resto de las variables se calculó a través del tamaño del efecto para datos no paramétricos. Diversos autores (Coe, 2002; Tomczak y Tomczak, 2014) recomiendan usar la correlación biserial por rangos de Glass (r), utilizando para ellos los rangos de la prueba de Mann-Whitney. Se obtuvieron los siguientes resultados: $r=.036$ para CL-ACL; .005 para CL-D; .18 para AL; .07 para SCL; y .02 para IF.

\section{Discusión y Conclusiones}

Nos propusimos averiguar, contestando a nuestro primer objetivo, cuáles eran las actitudes hacia la lectura (AL) y el sentimiento de competencia como lector (SCL) en el momento en que se está iniciando el aprendizaje de la lectura. La elevada media obtenida en el cuestionario indica que el alumnado de segundo curso de Educación Primaria afronta con actitudes muy positivas este aprendizaje, a pesar del esfuerzo que supone leer y comprender cuando aún no se han automatizado las correspondencias grafema-fonema. Asimismo, los participantes manifiestan un elevado sentimiento de competencia como lector (SCL), lo que no resulta extraño tras comprobar que hay una correlación de .50 entre esta variable y las AL. En ninguna de las dos variables hay diferencias en función del grado de implicación familiar en los procesos lectoescritores. Estos resultados coinciden con los reseñados en la literatura científica. Cuando los estudios se centran en los primeros cursos de Educación Primaria, nos dicen que ambas puntuaciones son altas (Izquierdo-Magaldi, Melero y Villalón, en prensa; Chapman y Tunmer, 1997; McGeown et al., 2015; Merisuo-Storm y Soininen, 2014), aunque en nuestro caso son más elevadas aún que las informadas por estos autores.

Respecto al segundo objetivo, hemos encontrado diferencias estadísticamente significativas entre niños y niñas en AL. Este resultado no es coincidente con el de Merisuo-Storm y Soininen (2014), quienes no las hallaron en el mismo curso de nuestro estudio (segundo de Educación Primaria) y se sitúa más en la línea del encontrado por McGeown et al. (2015) y por Artola et al. (2018) en ese mismo curso. También con lo que obtuvieron en cursos posteriores Mulllis et al. (2017) en cuarto, Logan y Johnston (2009) en quinto y Artola et al. (2017) 
en sexto, igual que Becker y McElvany (2018) desde tercero a sexto. Por su parte, otro estudio que se sitúa en segundo y que mide un aspecto cercano al de actitudes (motivación intrínseca hacia la lectura), el de Stutz, Schaffner y Schiefele (2016), informa de un tamaño del efecto de .14 , más bajo que el nuestro $(d=.33)$. Creemos que este resultado es relevante, a pesar de un tamaño del efecto pequeño, porque apunta a que las diferencias en España también empiezan a aparecer en los primeros cursos, aunque sea tímidamente. El estudio longitudinal de Becker y McElveny (2018) muestra, además, que van aumentando desde tercero a sexto. Asimismo, estas diferencias podrían irse ligando más al género que al sexo si nos atenemos a los resultados de McGeown (2015), con niños y niñas de 10 años, y de McGeown y Warhurst (2019) con sujetos de 9 a 11 años. Destacan que, más que el sexo, es la identidad de género la que predice la motivación lectora. Es importante, por tanto, que la lectura no se asocie al sexo, aunque tampoco, idealmente, al género femenino. En este sentido, sería importante que tanto padres como madres mostrasen con su ejemplo que la lectura es una actividad instrumental y de disfrute tanto para hombres como para mujeres, para que niños y niñas dispusiesen de ambos modelos. Aunque en nuestro estudio y en otros las diferencias entre niños y niñas en actitudes hacia la lectura sean pequeñas en los comienzos de la escolarización, el hecho de que sean tan tempranas junto con que se pudieran ir ligando más al género que al sexo lo convierte en un asunto educativo de primer orden. En lo que se refiere a SCL, no hemos hallado diferencias significativas entre niños y niñas, un resultado que aparece, por lo general, en la literatura existente (Logan y Johnston, 2009; Marinak y Gambrell, 2010; McGeown et al., 2015), aunque por el estudio de Becker y Elvany (2018) sabemos que pueden ir apareciendo durante la Educación Primaria. No obstante, Artola et al. (2018) encuentran diferencias significativas en SCL a favor de los niños ya en segundo de Educación Primaria.

Dentro también del segundo objetivo estaba averiguar si había diferencias en CL, AL y SCL en función de los tres grupos de implicación familiar (IF) (baja, media y alta). Hemos encontrado que estas diferencias son significativas respecto a la comprensión lectora, tanto si es medida por una prueba estandarizada (ACL), como si es valorada por el docente. Sin embargo, no son significativas en el caso de AL y SCL. En futuras investigaciones podría comprobarse si el impacto de IF sobre CL es directo o, por el contrario, se trata de un impacto indirecto o mediado a través de las AL o del SCL. El reciente estudio de Xia y Gu (2019) aborda precisamente esta cuestión. 
En cuanto al tercer objetivo, las relaciones entre las variables que hemos considerado (AL, SCL e IF en los procesos lectores que tienen lugar en la escuela) y la CL (estimada con dos medidas), hemos encontrado cuatro resultados que nos parece interesante comentar y que responden a nuestra última pregunta.

En primer lugar, tampoco hemos encontrado correlaciones entre AL y las medidas de CL, resultado que coincide con lo que sucedió en la investigación de Merisuo-Storm y Soininen (2014), en la de un estudio longitudinal, el de Nurmi y Aunola (2005) con niños de primero y segundo curso y, asimismo, en la de Mora-Figueroa et al. (2016). En cambio, nuestros datos no corroboran los de Chapman y Tunmer (1997), que encontraron en segundo curso una temprana relación entre CL y una escala de AL y SCL. Hay que tener en cuenta que, en nuestro caso, las altas puntuaciones obtenidas por prácticamente todos los participantes en AL impiden obtener correlaciones al no haber variabilidad en las puntuaciones.

En segundo lugar, a pesar de que es una correlación baja (.20) podemos decir que, en cambio, en nuestra muestra el autoconcepto lector, medido a través de la percepción de facilidad de la tarea, y la CL (evaluada por los docentes) están relacionados. Investigaciones anteriores que han encontrado este mismo resultado son la de Merisuo-Storm y Soininen (2014) en segundo y la de McGeown et al. (2015) en primero. En este sentido, creemos que desde la escuela debe hacerse un esfuerzo para que la complejidad progresiva que el alumnado va a encontrar en las tareas de CL no haga disminuir este autoconcepto lector, base motivacional o emocional del rendimiento lector.

En tercer lugar, se ha encontrado una correlación que merece tomarse en consideración entre IF y la medida de CL medida a través de una prueba estandarizada. Asimismo, esta correlación aumenta y se convierte en moderada cuando la medida de CL son las valoraciones hechas por el docente. Nuestros resultados respecto a IF son similares a los hallados en otros estudios, que encuentran que tiene relación con el rendimiento escolar desde Educación Infantil y, específicamente, con rendimiento lector a lo largo de toda la Educación Primaria. En nuestro caso, las correlaciones que hemos obtenido son más elevadas que las encontradas en los metanálisis revisados y apoyan los resultados de la metasíntesis de Wilder (2014). Por consiguiente, sería una estrategia adecuada en los centros escolares trabajar para que las familias otorguen a la comprensión lectora la importancia que merece como base de la mayoría de los aprendizajes escolares. El contexto familiar puede hacer mucho para que sus hijos desarro- 
llen una buena AL, desde estar al tanto de los procesos lectoescritores que tienen lugar en el colegio para apoyarlos hasta llevarlos a bibliotecas, pasando por regalarles libros, por ejemplo.

En cuarto lugar, respecto a la relación entre IF y AL y entre IF y SCL, no hemos encontrado relación entre ellas y tampoco ningún estudio con las características del nuestro para poder comparar adecuadamente este resultado. En cambio, merece la pena resaltar que IF es la única variable que ha correlacionado con las dos medidas de CL, relación que no se ha obtenido en nuestro caso con la variable de AL. A juzgar también por los resultados de la literatura precedente, vemos que la IF desempeña un papel más claro en la CL que las propias AL de los estudiantes. Creemos que esto es algo que las autoridades educativas y los centros escolares deberían tener en cuenta. Las primeras deberían apoyar a los segundos en las acciones sistemáticas y planificadas dirigidas a que las familias adquieran un compromiso con los aprendizajes lectoescritores que están llevando a cabo sus hijos e hijas. Asimismo, sería importante que el profesorado tuviese en cuenta las distintas preferencias de niños y niñas y tratase de darles respuesta para fomentar un desarrollo positivo de los aspectos motivacionales de la lectura (Merisuo-Storm, 2014; McGeown et al., 2012, 2016). Creemos que aquí hay una importante cuestión para el debate. Nos preguntamos si sería más adecuado que el profesorado fuese respetuoso con estas preferencias de género y permitiera que fueran los propios niños y las propias niñas quienes eligieran sus lecturas o, por el contrario, y desde un punto de vista educativo, resultaría más conveniente que los docentes pusieran en marcha estrategias activas para moldear dichas preferencias, de modo que niños y niñas pudieran enriquecer sus iniciales intereses espontáneos, con el objetivo de superar así los estereotipos de género.

En todo caso podemos concluir señalando que es posible, desde el sistema educativo, actuar para mejorar la CL desde el momento inicial, aprovechando las AL positivas y el elevado SCL hacia este aprendizaje que tiene el alumnado. Además de la actuación directa, es decir, la que centros y docentes pueden llevar a cabo a través de los planes de animación a la lectura y de la metodología basada científicamente para enseñar a leer y comprender con eficacia y sentido, cabe otra actuación indirecta, que es la de promover la implicación de las familias en este tipo crucial de aprendizaje. Estudios como el de Xia y Gu (2019) apuntan en la misma dirección, pues han hallado que la implicación de los padres tiene un efecto tanto directo como indirecto (vía el autoconcepto como lector) sobre la motivación hacia la lectura y que este efecto es más positivo para los niños que para las niñas. Es obvio que esto exige, a su 
vez, el apoyo de las autoridades educativas a los centros y a los docentes para que pueda llevarse a cabo mediante la dotación de los recursos organizativos, materiales y personales necesarios.

Consideramos que el conjunto de nuestros resultados es de interés tanto para la investigación como para la práctica docente, pero es preciso señalar sus limitaciones. Estas tienen que ver, entre otras, con el hecho de que se trata de un estudio descriptivo y correlacional, por lo que no podemos establecer relaciones causales. Asimismo, cabría mejorar la forma de valorar la implicación familiar para que no descansara solo en la percepción del docente y para que contemplara aspectos concretos de la actuación de la familia en el ámbito de la lectura. De este modo, se podría medir a través de un autoinforme de los propios familiares. No obstante, a pesar de sus limitaciones, este trabajo es una valiosa contribución, pues es el primer estudio en España sobre la relación entre las actitudes, el sentimiento de competencia hacia la lectura, la comprensión lectora y la implicación familiar al comienzo de la Educación Primaria.

\section{Referencias}

Adamski, A., Fraser, B.J., \& Peiro, M.M. (2013). Parental involvement in schooling, classroom environment and student outcomes. Learning Environments Research, 16(3), 315-328. http://dx.doi.org/10.1007/s10984-012-9121-7.

Artola, T., Sastre, S., \& Barraca, J. (2017). Diferencias de género en actitudes e intereses lectores: una investigación con alumnos españoles de Primaria. Bordón, 69(1), 11-26. https://doi.org/10.13042/Bordon.2016.37925.

Artola, T., Sastre, S., \& Alvarado, J. M. (2018). Evaluación de las actitudes e intereses hacia la lectura: validación de un instrumento para lectores principiantes. European Journal of Education and Psychology, 11(2), 141-157.

Becker, M., \& McElvany, N. (2018). The interplay of gender and social background: A longitudinal study of interaction effects in reading attitudes and behaviour. British Journal of Educational Psychology, 88(4), 529-549.

Briñol, P., Falces, C., \& Becerra, A. (2007). Actitudes. En J.F. Morales, M. Moya, E. Gaviria e I. Cuadrado, (pp. 457-490). Psicología Social. Madrid: Mc Graw-Hill.

Carroll, J.M., \& Fox, A.C. (2017). Reading self-efficacy predicts word reading but not comprehension in both girls and boys. Frontiers in Psychology, 7(2056). https://doi.org/10.3389/fpsyg.2016.02056. 
Castro, M., Expósito-Casas, E., López-Martín, E., Lizasoain, L., Navarro-Asencio, E., \& Gaviria, J. L. (2015). Parental involvement on student academic achievement: A meta-analysis. Educational Research Review, 14, 33-46. https://dx.doi.org/10.1016/j.edurev.2015.01.002.

Catalá, M., Català, G., Monclús, R., \& Molina, E. (2001). Evaluación de la comprensión lectora. Pruebas ACL. Barcelona: Graó.

Coe, R. (2002). It's the effect size, stupid. What effect size is and why it is important. Paper presented at the British Educational Research Association Annual Conference, Exeter, 12-14 september.

Conradi, K., Jang, B.G., \& McKenna, M.C. (2014). Motivation terminology in reading research: A conceptual review. Educational Psychology Review, 26(1), 127-164. https://doi.org/10.1007/s10648-013-9245-z.

Chapman, J.W., \& Tunmer, W.E. (1995). Development of young children's reading self-concepts: An examination of emerging subcomponents and their relationship with reading achievement. Journal of Educational Psychology, 87(1), 154-167. https://dx.doi.org/10.1037/00220663.87.1.154.

Chapman, J.W., \& Tunmer, W.E. (1997). A longitudinal study of beginning reading achievement and reading self-concept. British Journal of Educational Psychology, 67(3), 279-291. https://dx.doi.org/10.1111/j.2044-8279.1997.tb01244.x

Desforges, C., \& Abouchaar, A. (2003). The impact of parental involvement, parental support and family education on pupil achievements and adjustment: A literature review. Research Report, $433,1-86$.

Fantuzzo, J., McWayne, C., Perry, M. A., \& Childs, S. (2004). Multiple dimensions of family involvement and their relations to behavioral and learning competencies for urban, low-income children. School Psychology Review, 33(4), 467-480.

Galindo, C., \& Sheldon, S.B. (2012). School and home connections and children's kindergarten achievement gains: The mediating role of family involvement. Early Childhood Research Quarterly, 27(1), 90-103. http://dx.doi.org/10.1016/j.ecresq.2011.05.004.

Gonzalez-DeHass, A., Willems, P., \& Holbeinet, M.D. (2005). Examining the relationship between parental involvement and student motivation. Educational Psychology Review, 17(2), 99-123. https://dx.doi.org/10.1007/s10648-005-3949-7

Izquierdo-Magaldi, B., Melero, A., \& Villalón, R. (en prensa). Actitudes y sentimiento de competencia hacia la lectura y escritura del alumnado de $1^{\circ}$ y $2^{\circ}$ de Educación Primaria. Revista Complutense de Educación.

Jeynes, W.H. (2005). A meta-analysis of the relation of parental involvement to urban elementary school student academic achievement. Urban Education, 40(3), 237-269. https://dx.doi.org/10.1177/0042085905274540.

Kalman, J. (2008). Beyond definition: central concepts for understanding literacy. International Review of Education, 54, 523-538. https://doi.org/10.1007/s11159-008-9104-1. 
Kaniuka, T.S. (2010). Reading achievement, attitude toward reading, and reading self-esteem of historically low achieving students. Journal of Instructional Psychology, 37(2), 184-189.

Katzir T., Lesaux N.K., \& Kim Y.S. (2009). The role of reading self-concept and home literacy practices in fourth grade reading comprehension. Reading and Writing 22(3), 261-276. https://dx.doi.org/10.1007/s11145-007-9112-8.

Kikas, E., Palarinen, E., Soodla, P., Peets, K., \& Lerkkanen, M-K. (2018). Associations between reading skills, interest in reading, and teaching practices in first grade. Scandinavian Journal of Educational Research, 62, 6, 832-849.

https://doi.org/10.1080/00313831.2017.1307272

Kintsch, W., \& Rawson, K. (2008). Comprehension. En M.J. Snowling y Ch. Hulme (eds.), The Science of Reading: A Handbook (pp. 211-226). Oxford: Blackwell Publishing Ltd.

Kloosterman, R., Notten, N., Tolsma, J., \& Kraaykamp, G. (2010). The effects of parental reading socialization and early school involvement on children's academic performance: A panel study of primary school pupils in the Netherlands. European Sociological Review, 27(3), 291306. https://doi.org/10.1093/esr/jcq007.

Larocque, M., Kleiman, I., \& Darling, S.M. (2011). Parental involvement: The missing link in school $\begin{array}{llll}\text { achievement. Preventing } & \text { School } & \text { Failure, } & 55(3),\end{array}$ https://dx.doi.org/10.1080/10459880903472876.

Lockwood, M. (2012). Attitudes to reading in English primary schools. English in Education, 46(3), 228-246. https://dx.doi.org/10.1111/j.1754-8845.2012.01132.x.

Logan, S., \& Johnston, R. (2009). Gender differences in reading and attitudes: examining where these differences lie. Journal of Research in Reading, 3(2), 199-214. http://dx.doi.org/10.1111/j.1467-9817.2008.01389.x

Marinak, B. A., \& Gambrell, L. B. (2010). Reading motivation: Exploring the elementary gender gap. Literacy Research and Instruction, 49(2), 129-141.

McGeown, S. P. (2015). Sex or gender identity? Understanding children's reading choices and motivation. Journal of Research in Reading, 38(1), 35-46. https://doi.org/10.1111/j.1467-9817.2010.01481.x

McGeown, S., Goodwin, H., Henderson, N., \& Wright, P. (2012). Gender differences in reading motivation: does sex or gender identity provide a better account? Journal of Research in Reading, 35(3), 328-336. https://doi.org/10.1111/j.1467-9817.2010.01481.x

McGeown, S., Johnston, R.S., Walker, J., Howatson, K., Stockburn, A., \& Dufton, P. (2015). The relationship between young children's enjoyment of learning to read, reading attitudes, confidence and attainment. Educational Research, 57(4), 389-402. https://dx.doi.org/10.1080/00131881.2015.1091234 
McGeown, S., Osborne, C., Warhurst, A., Norgate, R., \& Duncan, L. (2016). Understanding children's reading activities: reading motivation, skill and child characteristics as predictors. Journal of Research in Reading, 39(1), 109-125.

https://dx.doi.org/10.1111/1467-9817.12060.

McGeown, S. P., \& Warhurst, A. (2019). Sex differences in education: exploring children's gender identity. Educational Psychology, 1-17. https://doi.org/10.1080/01443410.2019.1640349

McNamara, D. S., \& Magliano, J. (2009). Toward a comprehensive model of comprehension. In B. Ross (ed.), The Psychology of Learning and Motivation, vol. 51, (pp. 297-384), Burlington: Academic Press.

Merisuo-Storm, T. \& Soininen, M. (2012). Young boys' opinions about reading, literacy lessons and their reading competence. ICERI2012 Proceedings, 4109-4118.

Merisuo-Storm, T., \& Soininen, M. (2014). The interdependence between young students reading attitudes, reading skills, and self-esteem. Journal of Educational and Social Research, 4(2), 122-130. http://dx.doi.org/10.5901/jesr.2014.v4n2p122.

Mol, S., \& Bus, A. (2011). To read or not to read: A meta-analysis of print exposure from infancy to early adulthood. Psychological Bulletin, 137, 267-96. http://dx.doi.org/10.1037/a0021890.

Mora-Figueroa, J., Galán, A., \& López-Jurado, M. (2016). Efectos de la implicación familiar en estudiantes con riesgo de dificultad lectora. Ocnos, 15, 7-21. https://doi.org/10.18239/ocnos_2016.15.1.866.

Mullis, I.V., Martin, M.O., Foy, P., \& Hooper, M. (2017). PIRLS 2016. International Results in Reading. TIMSS \& PIRLS International Study Center, Lynch School of Education, Boston College and International Association for the Evaluation of Educational Achievement.

Nurmi, J. E., \& Aunola, K. (2005). Task-motivation during the first school years: A person-oriented approach to longitudinal data. Learning and Instruction, 15(2), 103-122. https://dx.doi.org/10.1016/j.learninstruc.2005.04.009.

Olivares, F., Fidalgo, R., \& Torrance, M. (2016). Differences in reading self-efficacy between school years and according to gender. Revista de Psicodidáctica, 21(1), 45-63.

Perfetti, C. A., \& Adlof, S. M. (2012). Reading comprehension: A conceptual framework from word meaning to text meaning. En Sabatini, J., Albro, E., y O'Reilly, T. (eds.), Measuring up: Advances in how we assess reading ability, (p. 3-20). Maryland: Rowman and Littlefield Education.

Petscher, Y. (2010). Meta-analysis of the relationship between attitudes towards reading and achievement in reading. Journal of Research in Reading, 33(4), 335-355. http://dx.doi.org/10.1111/j.1467-9817.2009.01418.x. 
Schiefele, U., Schaffner, E., Möller, J., \& Wigfield, A. (2012). Dimensions of reading motivation and their relation to reading behavior and competence. Reading Research Quarterly, 47(4), 427463. http://dx.doi.org/10.1002/RRQ.030.

Sénéchal, M., \& Young, L. (2008). The effect of family literacy interventions on children's acquisition of reading from kindergarten to grade 3: A meta-analytic review. Review of Educational Research, 78(4), 880-907. http://dx.doi.org/10.3102/0034654308320319.

Stutz, F., Schaffner, E., \& Schiefele, U. (2016). Relations among reading motivation, reading amount, and reading comprehension in the early elementary grades, Learning and Individual Differences, 45, 101-113. https://doi.org/10.1016/j.lindif.2015.11.022

Tomczak, M., \& Tomczak, E. (2014). The need to report effect size estimates revisited. An overview of some recommended measures of effect size. Trends in Sport Sciences, 1(21), 19-25.

Van Voorhis, F.L., Michelle F. Maier, M.F., Epstein, J.L., \& Lloyd, C.M. (2013). The impact of family involvement on the education of children ages 3 to 8 . New York: MDRC.

Wilder, S. (2014). Effects of parental involvement on academic achievement: a meta-synthesis. Educational Review, 66(3), 377-397. https://dx.doi.org/10.1080/00131911.2013.780009.

Xia, T., \& Gu, H. (2019). Effect of parents' encouragement on reading motivation: The mediating effect of reading self-concept and the moderating effect of gender. Frontiers in Psychology, 10, 609. https://doi.org/10.3389/fpsyg.2019.00609

Xu, M., Benson, S. N. K., Mudrey-Camino, R., \& Steiner, R. P. (2010). The relationship between parental involvement, self-regulated learning, and reading achievement of fifth graders: A path analysis using the ECLS-K database. Social Psychology of Education, 13(2), 237-269. https://doi.org/10.1007/s11218-009-9104-4 
Anexo I: Adaptación del cuestionario de Merisuo-Storm y Soininen (2012)

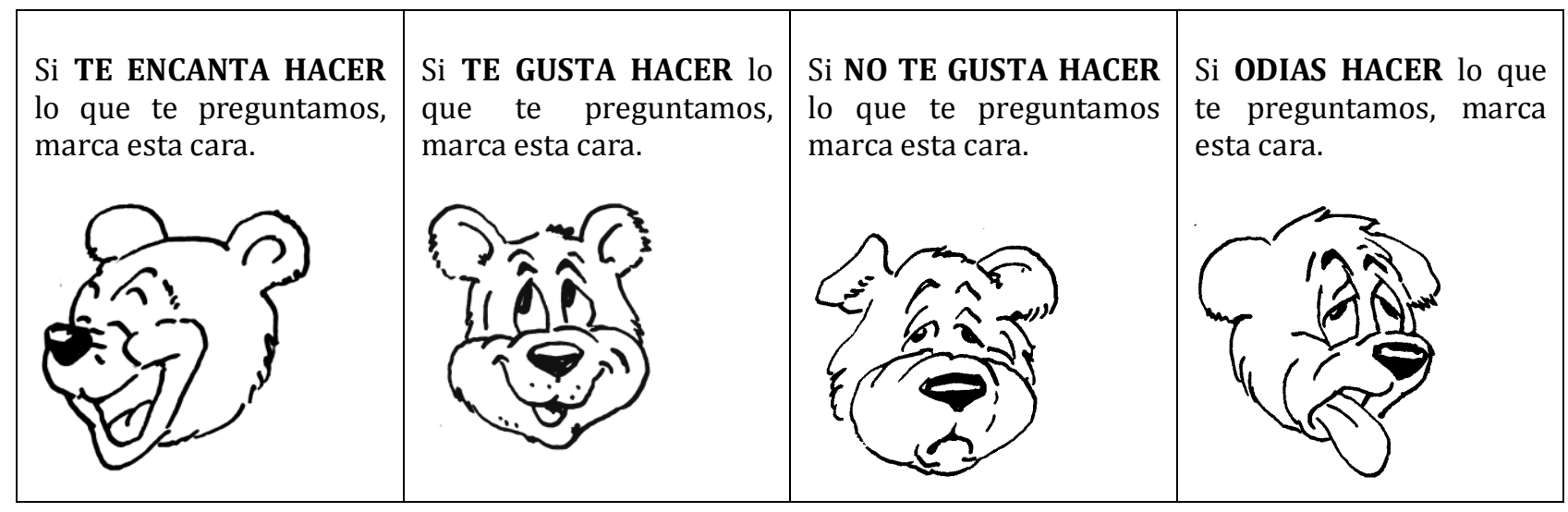

1. ¿Te gusta leer libros?

2. ¿Te gusta escribir?

3. ¿Te gusta leer cómics y/o tebeos?

4. ¿Te gusta que te regalen libros?

5. ¿Te gusta que te lean libros en voz alta?

6. ¿Te gusta leer cuentos?

7. ¿Te gusta leer libros que digan cómo son las cosas: animales, deportes, etc.?

8. ¿Te gusta ir a la biblioteca?

9. ¿Te gustó lo que se hace en el cole para aprender a leer?

10. ¿Te gustó lo que se hace en el cole para aprender a escribir?

11. ¿Te gustó leer esos libros que hay para aprender las letras?

12. ¿Te gustó hacer ejercicios para aprender a leer y a escribir?

13. ¿Te gusta escribir sobre lo que has leído?

14. ¿Te gusta hacer otras actividades sobre lo que has leído?

15. ¿Te gusta hacer los deberes de leer y escribir que tu profesor te manda para casa?

16. ¿Te gusta hablar con tus compañeros sobre los libros que estáis leyendo?

17. ¿Te gusta hacer los deberes de leer y escribir junto con algún compañero?

18. ¿Te gusta leer en voz alta en clase?

19. ¿Te gusta comentar con otros compañeros si te gustó un libro que has leído?

20. ¿Te gusta leer junto con otro compañero?

21. ¿Te resulta fácil leer?

22. ¿Te resulta fácil escribir?

23. ¿Has aprendido fácilmente a leer?

24. ¿Has aprendido fácilmente a escribir?

25. ¿Es fácil para ti entender los textos que lees en el colegio?

26. ¿Es fácil para ti entender las palabras que lees?

27. ¿Te resulta fácil acordarte de lo que lees?

Recibido: 09-10-2019

Aceptado: 03-02-2020 